\title{
Analysis of Multi-axes Adjustment Platform for Tunnel Intelligent Detection System
}

\author{
Xiao Liu ${ }^{1 *}$, Jie Zhang ${ }^{2}$, Erjuan Luo ${ }^{1}$, Menghua Liu ${ }^{3}$, Yingjie Duan ${ }^{1}$, Pengbo Li $^{3}$ and Chunming Xue ${ }^{1}$ \\ ${ }^{1}$ Key Lab of highway Construction \& Maintenance Technology in Loess Region, Shanxi Transportation Research Institute, \\ Taiyuan, Shanxi, People's Republic of China \\ ${ }^{2}$ Liren College of Yanshan University, Qinhuangdao, Hebei, People's Republic of China \\ ${ }^{3}$ Hebei Provincial Key Lab of Parallel Robot and Mechatronic System, Yanshan University, Qinhuangdao, Hebei, People's \\ Republic of China \\ *Corresponding author
}

\begin{abstract}
Based on the key technology problems of the tunnel intelligent detection system, this paper proposes a method of using multi-axes adjustment platform to solve the problem of image acquisition. The design method of multi-axes adjustment platform is proposed and a new type of 3-DOF parallel mechanism is designed. On the basis of kinematic analysis, the mapping between the input and output of the multi-axes adjustment platform is established, and the constraint conditions of workspace of the mechanism are defined. Finally, a design of servo control scheme and hardware selection of the experimental prototype is carried out. In order to test and validate the model, the experimental tests are put forward, and the workspace of the system is analyzed. This paper applies the robot technology to the field of machine vision, and offered a new method for developing tunnel intelligent detection system.
\end{abstract}

Keywords-multi-axes adjustment platform; 3-DOF parallel mechanism; mechanism model; tunnel rapid detection system

\section{INTRODUCTION}

Due to geological disasters, operating years and other reasons, highway tunnel will have cracks, which makes a serious threat to the operation safety of highway [1]. At present, tunnel crack detection means and equipment is relatively backward, people often using artificial or artificial instrument with high-altitude work vehicle way to complete the detection task. This method, based artificial detection, has low efficiency and big subjective degree, especially, our country has built a number of long tunnels. It cannot finish the detection task in the short term if rely on artificial. The latest release of "technical specifications for maintenance of highway tunnel" in 2015 pointed out: tunnel detection from "daily inspection" to "check usually", detection methods should used combination method of artificial and information means [2], which also shows that the rapid detection of intelligent equipment has become the need of the development of road tunnel maintenance.

With the CCD sensor technology and digital image processing technology is becoming mature, both at home and abroad have carried out research on rapid detection system based on machine vision [3-5], but the application in highway tunnel detection is relatively small, mostly at theoretical research and experimental stage. The core and key technology of machine vision is how to acquisition image high-quality [6], which requires the analyzed in the depth range of the camera. The structure of highway tunnel is complex, detection of the car driving the process inevitably cause surface relative pose changes of camera and lining, which also led to a decline in the quality of the collected images, seriously affect the work of subsequent image processing, character of crack identification. At present, people often choose the digital camera that has the low resolution and large depth of field, which can cause crack detection accuracy is low, so how to guarantee the high quality of the image acquisition is the key problem of rapid detection system of tunnel.

This passage bring the parallel robot technology into the field of machine vision, put forward a technical scheme which use multi axis adjusting platform to solve the difficulty in tunnel rapid detection system of image acquisition, and provides a new method and a new idea for the development of rapid detection system of tunnel.

\section{TunNEL InTELligent Detection System BASED ON MACHINE VISION}

\section{A. Working Principle}

The tunnel rapid detection system integrates digital camera, lens, light source, image acquisition card, data memory, inertial navigation device, distance sensor, generator and so on, form a set of vehicle detection system. In the process of vehicle driving, the tunnel lining surface is captured in real time, and the image data is transmitted to the memory through the data acquisition card, after the data analysis and processing, we can identify the characteristics of the cracks.

The system uses multiple cameras to collect half of the tunnel image, as shown in Fig.1. To achieve all the single tunnel detection through the two driving, to minimize the number and cost of hardware, while avoiding the 
surrounding vehicles on the image acquisition process interference.



FIGURE I. THE SCHEMATIC DIAGRAM OF MULTI CAMERA INSTALLATION

\section{B. Key Issues}

The depth of field is an important parameter of a digital camera, which is a pair of contradictory performance parameters with resolution. In order to ensure the accuracy of the crack, it need to choose the digital camera with high resolution, which also led to a small depth of field, in the collection process, it need to ensure the camera and lining surface distance strictly. But the test car at high speed have deviation inevitably influenced by the process of traffic flow, road conditions and driver's subjective consciousness, reduce the effect of camera, the quality of image acquisition is low, seriously affect the precision and accuracy of identification of fracture.

Secondly, highway tunnel structure is complex, different tunnel structure size will have big difference, so every time before the tunnel detection, it must according to the tunnel design drawings to adjust the camera installation position and focal length. On the one hand, the intelligent degree of the system is reduced. On the other hand, the vehicle bump affects the focusing device of the digital camera, which causes disturbance to the image acquisition.

In conclusion, in order to meet the requirements of the digital camera in shooting range and accuracy, it needs to be installed on a multi axis adjustable platform, according to the tunnel structure, vehicle trajectory deviation, it can make the real-time adjustment to the distance between camera and lining surface, and ensure the image collected by the cameras have overlapping. Parallel mechanism is easy to realize multi axis linkage, which has the characteristics of high precision and fast response [7-8], so it is an ideal mechanism model for developing multi axis adjustment platform of digital camera.

\section{Model Design AND Kinematic Characteristics ANAlysis of Multi AxIS AdJUSTMENT PlatForm}

\section{A. Models Design Principles}

The main types of motion of multi axis adjustment platform for digital camera are:
(1) The test vehicle in the running process make left and right swing motion, cause the changes of distance between the camera and the lining wall, therefore it need to have the ability to adjust the multi axis platform along the tunnel longitudinal movement, which keeps in the field depth range between the surface of the digital camera and the tunnel lining.

(2) The distance between the digital camera and the top of the tunnel is larger and the size of the tunnel structure is different, so the multi axis adjustment platform can adjust the vertical height of the digital camera.

(3) The multi axis adjustment platform compensates for the above two kinds of motion, the camera vision changes, resulting in too much overlap of images collected by multiple cameras, affecting subsequent processing. So the platform must have the ability to rotate around the direction of the vehicle, to ensure the camera can collect all the tunnel lining, and the collected images have overlapping parts.

Establish the coordinate system as shown in Fig.2, $\mathrm{X}$ axis along the vehicle forward direction (tunnel longitudinal), Y axis perpendicular to the lining surface, $Z$ axis vertical upward. From the above analysis, the multi axis adjustment platform can realize the rotational freedom around $\mathrm{X}$ axis and the freedom of movement along $\mathrm{Y}$ and $\mathrm{Z}$ axis, so a kind of three degree of freedom parallel mechanism is needed.

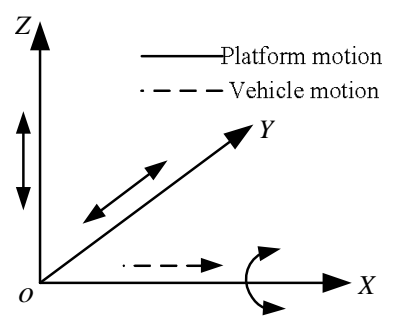

FIGURE II. THE DEGREE OF FREEDOM DIAGRAM OF MULTI AXIS ADJUSTMENT PLATFORM

The branch constraint system of the mechanism is three [9], and with only one binding. It containing a constraint couple and a binding, and it also can be containing two constraint couple and a binding. In order to ensure the multi axis adjusting platform can rotate continuously, the constraint screw which motor branch to mechanism should as little as possible, so this paper only contains a comprehensive and binding branch for five degrees of freedom configuration, all binding must satisfy the geometric conditions of parallel space and non coplanar.

\section{B. Design of Multi Axis Adjustment Platform Type}

Typical structures with branch degrees of freedom of five are RPS and SPR configurations, as shown in Fig.3. For RPS type branches, the coordinates of the moving helix in the system are: 


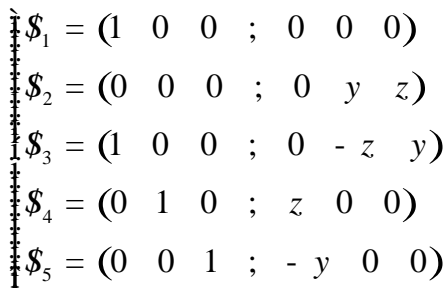

where, $\left(\begin{array}{lll}0 & y & z\end{array}\right)^{\mathrm{T}}$ is coordinate for the ball pair (S) center in the $\{o\}$ coordinate system.

The unique binding linear vector of the branch can be obtained from the upper form:

$$
\boldsymbol{\$}^{r}=\left(\begin{array}{lllllll}
1 & 0 & 0 & ; & 0 & -z & y
\end{array}\right)
$$

It represents the pivot point and parallel to the axis of rotation.

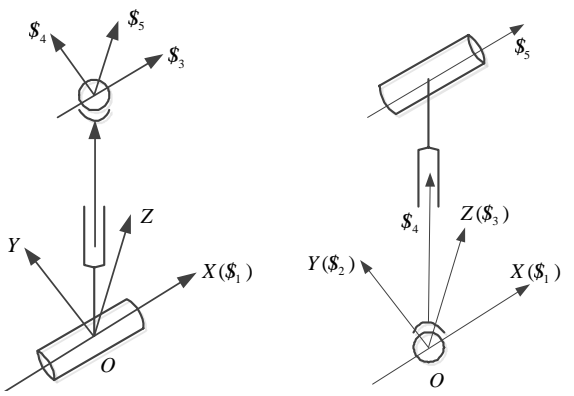

FIGURE III. THE CONSTRAINT SCREWS OF RPS AND SPR BRANCH

For the SPR bifurcation, the coordinates of the kinematic helix in the system are:

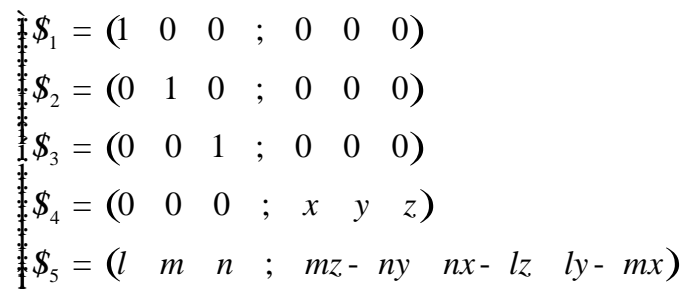

In the above equation, $\left(\begin{array}{lll}x & y & z\end{array}\right)^{\mathrm{T}}$ is coordinate of the rotation sub central point in the $\{o\}$ coordinate system. $\left(\begin{array}{lll}l & m & n\end{array}\right)^{\mathrm{T}}$ is the direction in axis of rotation, and satisfy the relationship $l x+m y+n z=0$.

Unique binding linear vector of the branch can be obtained from the upper form:

$$
\$^{r}=\left(\begin{array}{lllllll}
l & m & n & ; & 0 & 0 & 0
\end{array}\right)
$$

It represents the pivot point and parallel to the axis of rotation.

By (1) and (2), the following conclusions can be obtained: RPS or SPR branch of the binding line vector is always over the ball hinge point and parallel to the axis of rotation axis. Combined with the characteristics of the binding line vector of the parallel mechanism, the design of the multi axis adjustment platform is 2SPR/RPS parallel mechanism, as shown in Fig.4.

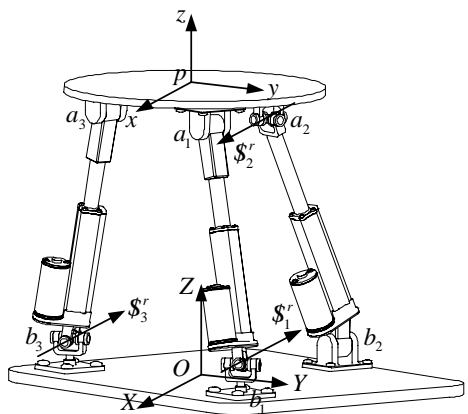

FIGURE IV. THE 2SPR/RPS PARALLEL MECHANISM

The 1st and 3rd branch is SPR configuration; the 2nd branch is RPS configuration, in which $\mathrm{P}$ is driven pair. The three moving branches are evenly distributed between the movable and the fixed platforms, and connected with the platform through corresponding motion pairs, and the corresponding hinge point centers are respectively. The $\mathrm{R}$ auxiliary axes of all branches are parallel to each other.

\section{Motion Feature Analysis}

When the mechanism at a initial position, the constraint screw system of the moving branch to the moving platform can be established by the binding (1) and (2):

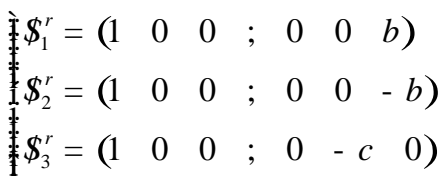

The 3 binding line vector does not satisfy the geometric conditions of coaxial, so the mechanism has no public constraint and redundant constraint, and the independent degrees of freedom is 3 .

The continuous rotation of physical existence of axes and mathematical criterion by the existence of rigid body rotation axis [10], in a single line vector constraints and binding line vector space parallel lines intersect at the point of the constraint may be taken as axis continuous rotation as a rigid body around its moving path; The moving path of a rigid body is perpendicular to the direction of all the constraint lines. For the moving platform constrained by 3 line vector constraints, and $\boldsymbol{\phi}_{1}^{r}, \boldsymbol{\$}_{2}^{r}$ and $\boldsymbol{\$}_{3}^{r}$ parallel to each other and are not coplanar, so the moving platform can realize continuous rotation about the $\mathrm{X}$ axis; $\mathrm{Y}$ axis and $\mathrm{Z}$ axis are perpendicular to $\boldsymbol{\$}_{1}^{r}, \boldsymbol{\$}_{2}^{r}$ and $\boldsymbol{\$}_{3}^{r}$, the moving platform can realize moving along $\mathrm{Y}$ and $\mathrm{Z}$ axis. 
The mechanism is analyzed in the kinematic characteristics of the initial pose, when institutions from the initial position to any position, we can obtain the kinematic characteristics of the same side. So the multi axis adjusting platform based on of 2SPR/RPS mechanism can realize tracking alignment of lateral displacement, lifting and roll of digital camera.

\section{Establishment OF MUlTi-AXES AdJUSTMENT PlATFORM MECHANISM MODEL}

\section{A. Mapping Relation of Input- Output \\ 1) Position the solution}

The description of each point of moving platform and fixed platform in the respective coordinate system is $\boldsymbol{a}_{i}^{p}, \boldsymbol{b}_{i}^{o}$, and the digital camera needs to adjust the amount of $\left(\begin{array}{lll}\alpha & y & z\end{array}\right)^{\mathrm{T}}$. The transformation matrix of the corresponding multi axis platform is $\mathrm{T}$ :

$$
\boldsymbol{T}=\left[\begin{array}{ccc}
0 & 0 & 0 \\
\cos \boldsymbol{\alpha} & \sin \boldsymbol{\alpha} & \boldsymbol{y} \\
\sin \boldsymbol{\alpha} & \cos \boldsymbol{\alpha} & z \\
0 & 0 & 1
\end{array}\right]
$$

The description of each hinge point of the moving platform in the $\{o\}$ system is $\overline{\boldsymbol{a}}_{i}^{0}$ :

$$
\overline{\boldsymbol{a}}_{i}^{0}=\boldsymbol{T}_{i}^{p}
$$

The driving branch of the mechanism is $\mathrm{P}$, and the displacement is the drive displacement of the mechanism. Combined with(4),(5) and multi axis adjustment platform geometric dimensions, the pose of the mechanism are established:

$$
q_{i}=\left[\begin{array}{lll}
q_{1} & q_{2} & q_{3}
\end{array}\right]=\left|a_{i}^{0}-b_{i}^{0}\right|
$$

where, $q_{i}$ is the movement displacement of each branch.

\section{2) Velocity and acceleration mapping}

The multi axis platform needs to provide the corresponding motion according to the speed and acceleration, so it is necessary to establish the mapping relationship between the velocity and acceleration of the driver. According to the adjustment of digital camera, the derivative of time is solved, the six dimensional velocity and acceleration expressions of the multi axis platform are expressed respectively:

$$
\begin{aligned}
& \grave{1=} \boldsymbol{v}=\left(\begin{array}{llllll}
\dot{\alpha} & 0 & 0 & 0 & \dot{y} & \dot{z}
\end{array}\right)^{\mathrm{T}} \\
& \stackrel{1}{\mathbf{1}} \boldsymbol{a}=\left(\begin{array}{llllll}
\ddot{\alpha} & 0 & 0 & 0 & \ddot{y} & \ddot{z}
\end{array}\right)^{\mathrm{T}}
\end{aligned}
$$

Based on the theory of parallel robot [11], the mapping relation of velocity and acceleration of the multi axis platform can be obtained as:

$$
\begin{aligned}
& \dot{\boldsymbol{q}}=\left[\begin{array}{lll}
\dot{q}_{1} & \dot{q}_{2} & \dot{q}_{3}
\end{array}\right]^{T}=\boldsymbol{G} \boldsymbol{v} \\
& \ddot{\boldsymbol{q}}=\left[\begin{array}{lll}
\ddot{q}_{1} & \ddot{q}_{2} & \ddot{q}_{3}
\end{array}\right]^{T}=\boldsymbol{G} \boldsymbol{a}+\boldsymbol{v}^{\mathrm{T}} \boldsymbol{H} \boldsymbol{v}
\end{aligned}
$$

where, $\mathrm{G}$ and $\mathrm{H}$ are the first and second order influence coefficient of mechanism; $\dot{q}_{i}$ and $\ddot{q}_{i}$ are the driving velocity and acceleration of each branch.

\section{B. Rotation and displacement working space}

For the 2SPR/RPS parallel mechanism, the constraint condition is mainly composed as the following parts:

(1) Driven constraints: the mechanism driven by the electric cylinder and other linear drivers, not beyond the safe range of the electric cylinder, the development of constraints for the:

$$
l_{i}^{\min } \# q_{i} \quad l_{i}^{\max }
$$

where, $l^{\min }$ and $l^{\max }$ are respective the minimum and maximum values of the electric cylinder stroke.

(2) Rotation angle constraint: Revolute pair is restricted by the rotation of the shaft, bearing and other components, so it is necessary to limit the rotation angle, to ensure that it is in the rotation range and does not interfere with the corresponding components of the dynamic and fixed platform. First, the calculation formula of the rotation angle is needed. By (4) and (5), the direction vector of the connecting rod aibi in the $\{o\}$ system is $\boldsymbol{m}_{\mathrm{i}}^{\circ}$ :

$$
\boldsymbol{m}_{i}^{o}=\left({ }^{o} \boldsymbol{a}_{i}-{ }^{o} \boldsymbol{b}_{i}\right) /\left|{ }^{o} \boldsymbol{a}_{i}-{ }^{o} \boldsymbol{b}_{i}\right|
$$

For the second branch, the revolute pair is connected with the fixed platform, and the angle of the revolute pair is described by the angle between the link direction vector and the $\{o\}$ unit vector $\lambda=\left[\begin{array}{lll}0 & 1 & 0\end{array}\right]^{\mathrm{T}}$ :

$$
\psi_{2}=\cos ^{-1}\left[\left(\boldsymbol{m}_{i}^{o} \cdot \lambda\right) /\left|\boldsymbol{m}_{i}^{o} \times \lambda\right|\right]
$$

Similarly, for the first and the third branches, the rotation pair is connected with the moving platform, and the rotation angle of the rotating pair is described by the direction vector 
of the connecting rod and the rotation angle of the $\{p\}$ unit vector $\lambda=\left[\begin{array}{lll}0 & 1 & 0\end{array}\right]^{\mathrm{T}}$ :

$$
\psi_{i}=\cos ^{-1}\left\{\left[m_{i} \cdot(\boldsymbol{R} \lambda)\right] /\left|m_{i} \times(\boldsymbol{R} \lambda)\right|\right\}, \quad(i=1,3)
$$

where, $\boldsymbol{R}$ is the transformation matrix of $\{o\}$ to $\{p\}$.

Based on the (11) and (12), the constraint condition of the rotation pair is formulated as:

$$
\psi_{i}^{\min } \# \psi_{i} \quad \psi_{j}^{\max }
$$

where, $\psi_{i}^{\min }$ and $\psi_{j}^{\max }$ are respectively the limit range of motion of revolute pair.

(3) Ball joint motion constraints: Globe hinge axis by concurrent in one point and the vertical each other three rotation, need by calculating the angle of globe hinge expression. The first branch is taken as an example, and the coordinate system of each link is shown in Fig.5.

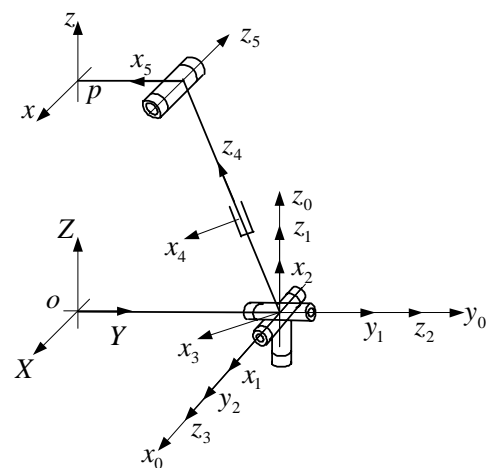

FIGURE V. THE COORDINATE SYSTEM OF THE $1{ }^{\text {ST }}$ BRANCH

According to the linkage parameters, the transformation matrix between the connecting rods is $\boldsymbol{T}_{1}^{0}, \boldsymbol{T}_{2}^{1}, \boldsymbol{T}_{3}^{2}, \boldsymbol{T}_{4}^{3}, \boldsymbol{T}_{5}^{4}$, $\boldsymbol{T}_{p}^{5}$,it can be obtained as $\boldsymbol{T}_{4}^{0}=\left(\boldsymbol{T}_{0}^{o}\right)^{-1} \boldsymbol{T}_{p}^{o}\left(\boldsymbol{T}_{p}^{5}\right)^{-1}\left(\boldsymbol{T}_{5}^{4}\right)^{-1}$. When the motion parameters of the platform are known, the value of $\boldsymbol{T}_{4}^{0}$ can be obtained according to the inverse solution of position and attitude, assume:

$$
\boldsymbol{T}_{4}^{0}=\left(\boldsymbol{T}_{0}^{o}\right)^{-1} \boldsymbol{T}_{p}^{o}\left(\boldsymbol{T}_{p}^{5}\right)^{-1}\left(\boldsymbol{T}_{5}^{4}\right)^{-1}=\left[\begin{array}{cccc}
n_{x} & o_{x} & a_{x} & p_{x} \\
n_{y} & o_{y} & a_{y} & p_{y} \\
n_{z} & o_{z} & a_{z} & p_{z} \\
0 & 0 & 0 & 1
\end{array}\right]
$$

The following relations can be established by the transformation matrix:

$$
\boldsymbol{T}_{4}^{1}=\boldsymbol{T}_{2}^{1} \boldsymbol{T}_{3}^{2} \boldsymbol{T}_{4}^{3}=\left[\begin{array}{cccc}
\mathrm{c} \theta_{2} \mathrm{c} \theta_{3} & -\mathrm{c} \theta_{2} \mathrm{~s} \theta_{3} & -\mathrm{s} \theta_{2} & 0 \\
0 & 0 & \mathrm{c} \theta_{3} & \mathrm{c} \theta_{3} d_{4} \\
-\mathrm{s} \theta_{2} \cos \theta_{3} & \mathrm{c} \theta_{2} & \mathrm{~s} \theta_{2} \mathrm{~s} \theta_{3} & -\mathrm{s} \theta_{2} \mathrm{~s} \theta_{3} d_{4} \\
0 & 0 & 0 & 1
\end{array}\right]
$$

$$
\begin{aligned}
& \boldsymbol{T}_{4}^{1}=\left[\boldsymbol{T}_{1}^{0}\left(\theta_{1}\right)\right]^{-1} \boldsymbol{T}_{4}^{0} \\
& =\left[\begin{array}{cccc}
\mathrm{c} \theta_{1} n_{x}+\mathrm{s} \theta_{1} n_{y} & \mathrm{c} \theta_{1} o_{x}+\mathrm{s} \theta_{1} o_{y} & \mathrm{c} \theta_{1} a_{x}+\mathrm{s} \theta_{1} a_{y} & \mathrm{c} \theta_{1} p_{x}+\mathrm{s} \theta_{1} p_{y} \\
-\mathrm{s} \theta_{1} n_{x}+\mathrm{c} \theta_{1} n_{y} & -\mathrm{s} \theta_{1} o_{x}+\mathrm{c} \theta_{1} o_{y} & -\mathrm{s} \theta_{1} a_{x}+\mathrm{c} \theta_{1} a_{y} & -\mathrm{s} \theta_{1} p_{x}+\mathrm{c} \theta_{1} p_{y} \\
n_{z} & o_{z} & a_{z} & p_{z} \\
0 & 0 & 0 & 1
\end{array}\right]
\end{aligned}
$$

where, $\theta_{1}, \theta_{2}, \theta_{3}$ are respectively the rotation angle of the three revolute joints of the ball hinge, and $d_{4}$ is the driving force.

The two ends of (14) and (15) has the relationship as:

$$
\left\{\begin{array}{l}
\mathrm{c} \theta_{1} n_{y}-\mathrm{s} \theta_{1} n_{x}=0 \Rightarrow \theta_{1}=\tan ^{-1}\left(n_{y} / n_{x}\right) \\
\mathrm{c} \theta_{2}=o_{z} \Rightarrow \theta_{2}=\cos ^{-1}\left(o_{z}\right) \\
\mathrm{c} \theta_{1} a_{y}-\mathrm{s} \theta_{1} a_{x}=\mathrm{c} \theta_{3} \Rightarrow \theta_{3}=\cos ^{-1}\left(-\mathrm{s} \theta_{1} a_{x}+\mathrm{c} \theta_{1} a_{y}\right)
\end{array}\right.
$$

Similarly, the expression of the other branches of the ball hinge can be established:

$$
\theta_{i j}^{\min } \# \theta_{i j} \quad \theta_{i j}^{\max },(i=1,2,3 ; j=1,2,3)
$$

where, $\theta_{i j}^{\min }$ and $\theta_{i j}^{\max }$ are the rotation angle limit of revolute pair respectively.

\section{V.PROTOTYPE AND EXPERIMENT OF MULTI AXIS ADJUSTMENT PLATFORM}

\section{A. Hardware Selection and Control Scheme}

For structure parameters of multi axis adjustable platform: hinge point of moving platform is circumscribed circle diameter $d_{a}=1000 \mathrm{~mm}$, fixed platform hinge point diameter: $d_{b}=1200 \mathrm{~mm}$, initial height: $h=1000 \mathrm{~mm}$.

The servo motor for MITSUBISHI low inertia series, rated power $200 \mathrm{~W}$, rated speed $3000 \mathrm{r} / \mathrm{min}$, rated torque $0.64 \mathrm{Nm}$; The accuracy of the 131072 pulses/rev encoder; Electric cylinder stroke $1200 \mathrm{~mm}$; Using three axis gyro, accelerometer measurement to three combinations of moving platform pose parameters, detection accuracy of 1 degrees/sec gyroscope, accelerometer detection accuracy of $40 \mathrm{mg}$; Using Advantech PCI-1265-AE multi axis motion control card to 
achieve synchronous control and signal acquisition of the three branches.

The control flow of the platform is as follows: the relative position between the digital camera and the lining surface is collected by the laser scanner, which is converted to the active motion of the multi axis platform. Based on the input-output mapping relationship to calculate the displacement, velocity and acceleration, the multi axis motion control card to control the synchronous servo motor, encoder of branch of mobile, as feedback signal and real-time compensation of motor. At the same time, the real-time position and attitude of the gyro and accelerometer are compared with the theoretical value, and the motor motion is corrected in real time.

\section{B. Test and Analysis}

\section{1) Analysis of input-output mapping}

In order to make the research more general, we assume that the motion law of the digital camera is adjusted by the external disturbance:

$\alpha(t)=A_{\alpha} \sin \left(\omega_{\alpha} t\right), \quad y(t)=A_{y} \sin \left(\omega_{y} t\right), \quad z(t)=A_{z} \sin \left(\omega_{z} t\right)$

where, $A_{\alpha}=5^{\circ}, A_{y}=300 \mathrm{~mm}, A_{z}=400 \mathrm{~mm}$ are the degrees of freedom; $\omega_{\alpha}=\omega_{z}=\pi / 5 \mathrm{rad} / \mathrm{s}, \omega_{y}=\pi / 2$ are the degrees of freedom angular frequency.

Combined with the structure parameters and the input-output mapping relationship ((4) (8)), the displacement and velocity of each drive branch can be calculated, as shown in Fig.6. The motion law is taken as the target value of the test prototype, and the real-time curves of displacement and velocity of each electric cylinder are read through the multi axis motion control card, as shown in Fig 7. Fig.7. Compared with Fig.6 and Fig.7, theoretical calculation curve and experimental curve are consistent, which verifies the correctness of theoretical modeling. The real-time curve of continuous change, which also shows that the proposed 2SPR/RPS type multi axis adjustable platform motion performance is good, can satisfy the practical application requirements.

\section{2) The workspace analysis of the corner displacement}

In practical application, the adjustment of the inclination angle of the multi axis adjustment platform is relatively small, so a number of typical side slopes can be selected, which can be used to describe the two degrees of freedom of the work space. Select roll angle is divided into $0^{\circ}, 10^{\circ}, 20^{\circ},-20^{\circ}$ and based on the input-output mapping relationship ((4) (8)) and constraints ((9), (13), (17)), draw the working space of the platform, as shown in Fig.8 .Whether the platform can achieve a set of lateral and lift movement, only to see whether the corresponding coordinates fall in the shadow.

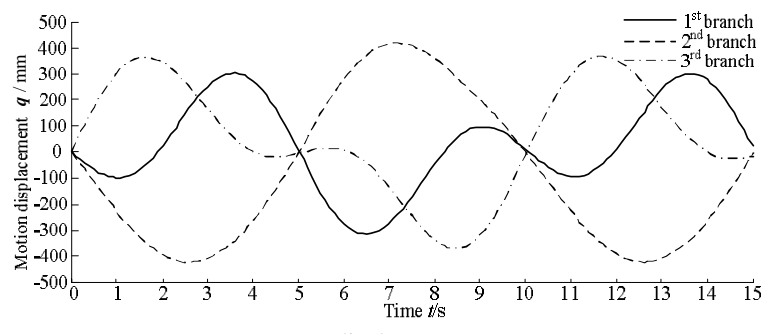

(a) displacement

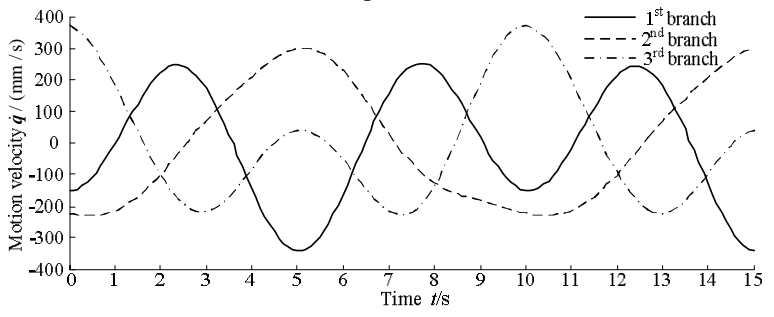

(b) velocity

FIGURE VI. THE CALCULATION CURVES OF EACH DRIVE BRANCH

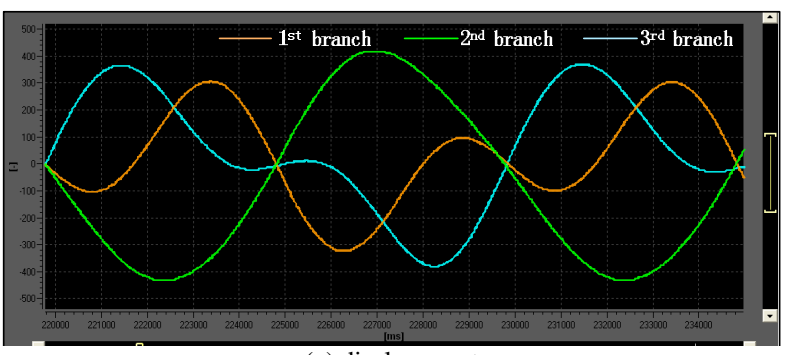

(a) displacement

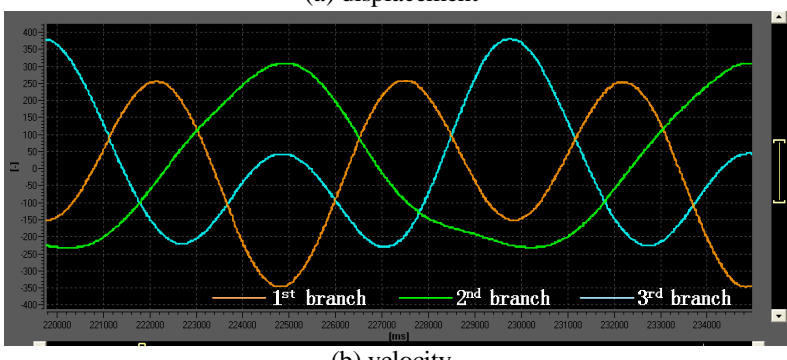

(b) velocity

FIGURE VII. EXPERIMENTAL CURVES OF EACH BRANCH

For the graph (a), the working space in a straight line with $y=0$ symmetric distribution, when $z=-93 \mathrm{~mm}$ roll capacity can reach the maximum value, it can be highly optimized for this platform initial position, namely $h=(1000-93) \mathrm{mm}=907 \mathrm{~mm}$; Comparison (a) (c), with the side angle increasing, the size of the workspace decreases, platform movement ability change difference; comparison chart (c) (d), side angle are equal and opposite, the size of the workspace is basically the same, but the movement characteristics in the working space is completely opposite. 


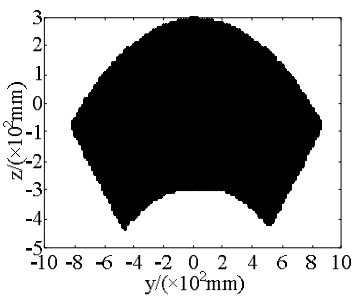

(a) $\alpha=0^{\circ}$

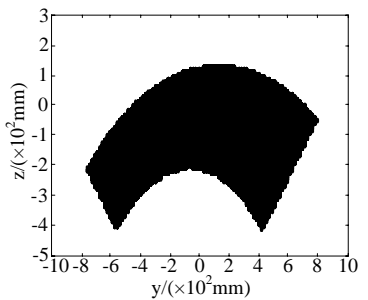

(c) $\alpha=20^{\circ}$

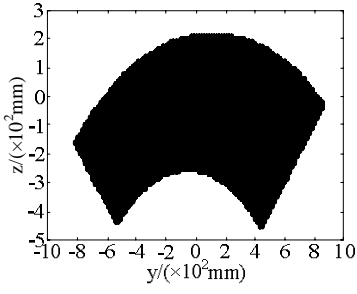

(b) $\alpha=10^{\circ}$

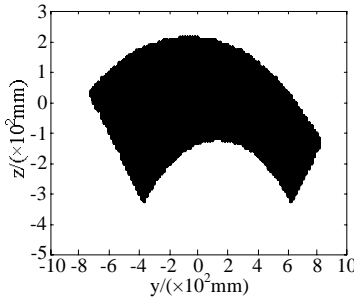

(d) $\alpha=-20^{\circ}$
FIGURE VIII. THE CORRESPOND WORKSPACE OF DIFFERENT ROLL ANGLE

\section{CONCLUSION}

(1) The parallel robot technology into the field of machine vision, put forward a multi axis adjusting platform to solve the tunnel rapid detection system of image acquisition difficult technical scheme, and provides a new method and a new idea for the development of rapid detection system of tunnel.

(2) Through the analysis of the characteristic of sports organizations, proved that the new $1 \mathrm{R}^{\mathrm{x}} 2 \mathrm{~T}^{\mathrm{yz}}$ design of three DOF parallel mechanism can meet the requirements of digital camera pose adjustment, is a feasible vehicle with multi axis adjustable platform type.

(3) Establish and define a platform for transmission into the output mapping relationship and work space constraints, design and hardware selection and servo control scheme of test prototype.

(4) The test results of the prototype show that the calculation results agree well with the experimental results, proved the correctness of theoretical modeling; the real-time curve of continuous change, no mutation, which also shows that the 2SPR/RPS design of multi axis motion control platform has good performance and can meet the practical requirements should be used.

From the theoretical level, the working space of the platform is analyzed. The analysis results provide the basis for the optimization of the structural parameters of the multi axis platform and the rationality of the adjustment of the camera motion.

\section{ACKNOWLEDGEMENT}

This research is sponsored by the National Natural Science Foundation of China (grant no. 51705229), National Natural Science Foundation of Shanxi Province (grant no.
2015021126) and Science Research Project of Shanxi Transportation Department (grant no.2017-1-25).

\section{REFERENCES}

[1] J. Ma, S. Z. Sun, and W. Y. Zhao, Raw on China's tunnel engineering research, China Journal of Highway and Transport, vol. 28(5), pp. 5-69, 2015 .

[2] JTG H12-2015,Technical specification of maintenance for highway tunnel[S].

[3] X. Wang, P. Wang, H. Cheng, Research of bridge displacement on-line monitoring technique based on machine vision, Journal of Highway Engineering, vol. 39(1), pp. 209-212, 2015.

[4] Y. D. Wang, Z. J. Yu, B. Bai, Research on image processing based subway tunnel crack identification algorithm, Chinese Journal of Scientific Instrument, vol. 35(7), pp. 51-58, 2015.

[5] Y.C. Song, G. X. Yan, Y. Q. Su, Texture structure distribution of asphalt pavement surface based on digital image processing technology, Journal of Central South University, vol. 45(11), pp. 4075-4080, 2014.

[6] X. Z. Liu, K. Ye, A long-distance image measuring technique for crack on tunnel lining, Journal of Tongji University, vol. 40(6), pp. 27-34,2014.

[7] X. Liu, T. S. Zhao, E. J. Luo, Coupling 3-PSR/PSU 5-Axis compensation mechanism for stabilized platform and its analysis, Proceedings of the Institution of Mechanical Engineers, Part C: Journal of Mechanical Engineering Science, vol. 227(7), pp. 1619-1629, 2013.

[8] F. Z. Yuan, T. S. Zhao, Y. Z. Zhao, Analysis of load carrying capacity of parallel mechanism, China Mechanical Engineering, vol. (26), pp. 23-29, 2014.

[9] Q.C. Li, Type synthesis theory of lower-mobility parallel mechanisms and synthesis of new architectures. Qing Huangdao: Yanshan University, pp. 63-65, 2013.

[10] E. J. Luo, D. J. Mou, X. Liu, A 3-DOF coupling parallel mechanism for stabilized platform and its motion characteristics, Robot, vol. 32(5), pp. 107-113, 2013.

[11] Z. Huang, Y.S. Zhao, T. S. Zhao, Advanced Spatial Mechanism. Beijing: High Education Press, 2006. 